\title{
Envejecimiento activo, objetivos y principios: retos para el trabajo social
}

\author{
Ana Belén Cuesta Ruiz-Clavijo \\ Departamento de Derecho y Trabajo Social, Universidad de La Rioja \\ ana-belen.cuesta@unirioja.es
}

\begin{abstract}
Biztanleriaren zahartzea erronka da gizartearentzat, baina baita aldibereko aukera ere, bai heterogeneoa den biztanleria-talde horren premia sozialei erantzuteko, bai sozialki ekarpenak egiten dituzten faktore positiboak txertatzeko. Artikulu honek bi helburu ditu. Lehenengoa, gizarte-lanaren eta zahartze aktiboaren arteko loturan sakontzean datza. Horretarako, zahartze aktiboaren eta gizarte-lanaren determinatzaileak eta printzipioak hartzen dira erreferentziatzat, baita eskuartzemailak eta kolektibo profesional horrek betetzen dituen funtzioak ere; horretaz gain, espazio komunak eta bereizleak behatzen dira. Bigarrena, aurreko azterketatik ondorioztatua, gizartelaneko profesionalek zahartze aktiboa sustatzea ahalbidetzen dutenez, erronka profesionalak azaltzean datza.
\end{abstract}

\section{GAKO-HITZAK:}

Gizarte-lana, zahartze aktiboa, adineko pertsonak, printzipioak, mailak, funtzioak.
El envejecimiento de la población constituye un reto para la sociedad y al mismo tiempo una oportunidad, tanto para responder a las necesidades sociales de este grupo de población tan heterogéneo como para incorporar los factores positivos que aportan socialmente. Este artículo tiene una doble finalidad. La primera consiste en ahondar en la vinculación existente entre trabajo social y envejecimiento activo. Para ello se toman como referencia los determinantes y principios del envejecimiento activo y del trabajo social; los niveles de intervención y las funciones desempeñadas por este colectivo profesional, y se observan los espacios comunes y diferenciadores. La segunda consiste en exponer los retos profesionales que devienen del análisis anterior y permiten promover el envejecimiento activo por parte de los profesionales del trabajo social.

\section{Palabras Clave:}

Trabajo social, envejecimiento activo, personas mayores, principios, niveles, funciones. 


\section{Introducción}

El reto demográfico del envejecimiento de la población es un elemento recurrente en los textos de políticas sociales. Su percepción como oportunidad para la sociedad en general y para las personas mayores en particular, se reitera en la bibliografía que vincula envejecimiento activo y trabajo social, objeto de estudio de nuestro trabajo (Ludi, 2012; Min, 2013; Cordero del Castillo, 2012; Barbosa, Fernández y Rodríguez, 2015; Bellido, 2013; Marshall y Altpeter, 2005; Fernández-García y De León, 2013; Amy, 2014; De la Villa, 2017; Salmerón, 2012; Tiernes, 2011; Foster y Walker, 2015; Marsillas, 2016). Los autores coinciden al describir una sociedad con una tasa alta de envejecimiento, una longeva esperanza de vida y la tasa de natalidad más baja de la historia. Por todo ello, plantean la necesidad de incorporar el paradigma positivo de envejecimiento e integrarlo en las diversas políticas sociales.

Otra idea que aparece en los discursos se relaciona con la heterogeneidad de las personas mayores (Marsillas, 2016; Cordero del Castillo, 2012; São José, Timonen, Amado y Santos, 2017; Ludi, 2012; Bellido, 2013; Nieto, 2014; Foster y Boxall, 2015; Bosch, Gómez y Ferrer, 2009). Exponen los autores la exigencia de superar una visión uniforme y homogénea de este grupo de población y reconocerlos como una suma de personas diversas, quienes presentan situaciones variadas que requieren respuestas individualizadas. La heterogeneidad, la diversidad y las necesidades diferenciadas son cuestiones que impregnan los discursos que vinculan el envejecimiento activo y el trabajo social. Por todo ello es necesario que las acciones dirigidas a la promoción del envejecimiento activo sean diferenciadoras e individualizadas superando el concepto de "one size fitst alls", que propone las mismas estrategias de envejecimiento para toda la población mayor (São José et al., 2017).

En el trabajo que nos ocupa, partimos, en primer lugar, del concepto de envejecimiento activo que recoge la Organización Mundial de la Salud (OMS) en 2002 y su vinculación con la disciplina del trabajo social. Posteriormente analizamos los determinantes del envejecimiento activo con los objetivos del trabajo social y observamos cómo estos se entrelazan. A continuación, abordamos los principios que inspiran el envejecimiento activo y los del trabajo social tomando en consideración principalmente el código deontológico (FITS, 2018). Avanzamos en dicho análisis y consideramos los diferentes niveles de intervención individual, grupal y comunitaria en los que se desarrollan prácticas de envejecimiento activo desde el trabajo social y las funciones profesionales que tratan de promover el envejecimiento activo.
Una segunda parte del artículo recoge diversos retos para los profesionales que realizan intervenciones sociales con la finalidad de promover un envejecimiento activo.

\section{Objetivos}

Los objetivos de este artículo son los siguientes:

1. Abordar la vinculación entre la disciplina del trabajo social y el envejecimiento activo.

2. Presentar retos profesionales que respondan a las necesidades/oportunidades derivadas del envejecimiento de la población.

Como objetivos específicos se plantean los siguientes:

a. Relacionar los determinantes del envejecimiento activo con los objetivos del trabajo social.

b. Comparar los principios del envejecimiento activo con los de la disciplina de trabajo social.

c. Analizar los niveles de intervención en los que se desarrollan prácticas de envejecimiento activo desde el trabajo social.

d. Abordar las funciones profesionales de esta disciplina para promover el envejecimiento activo.

\section{Metodología}

La metodología empleada en esta investigación se basa en un análisis bibliográfico acerca del envejecimiento activo y trabajo social. La búsqueda se realiza en la base de datos Ebsco Discovery (EDS). Los criterios son referencias que vinculen trabajo social y envejecimiento activo posteriores al año 2000 y limitadas a Europa. Esta búsqueda se realiza en julio 2018. La mayoría de ellas son artículos, aunque también se pueden encontrar actas de congresos y trabajos de fin de grado. Se realiza también la búsqueda de tesis en la base de datos de Teseo; si bien es cierto que existen veinticuatro tesis que tienen en su título "envejecimiento activo", encontramos fuera de dicha base de datos una que aborda directamente ambas cuestiones (Ahmed, 2013).

Agrupamos los textos revisados atendiendo a sus contenidos principales. A continuación, se exponen los principales hallazgos relacionados con la vinculación existente entre ambos elementos, teniendo en cuenta los objetivos, principios, niveles de intervención, funciones de los profesionales del trabajo social y el concepto del envejecimiento activo. 


\section{Entrelazando el trabajo social y el envejecimiento activo}

\section{El envejecimiento activo es definido por la OMS como:}

El proceso de optimización de las oportunidades de salud, participación y seguridad, con el fin de mejorar la calidad de vida a medida que las personas envejecen [...] es el proceso que permite a las personas realizar su potencial de bienestar físico, social y mental a lo largo de todo su ciclo vital y participar en la sociedad de acuerdo con sus necesidades, deseos y capacidades, mientras que les proporciona protección, seguridad, y cuidados adecuados cuando necesitan asistencia. (OMS, 2002: 79)

Este concepto presenta un objetivo más amplio que otros elementos como envejecimiento saludable, productivo y exitoso, que son parciales y reduccionistas, ya que enfocan a una o varias dimensiones como la funcional, la laboral o la participativa y ponen el punto de mira en el individuo como único responsable de su proceso de envejecimiento. El envejecimiento activo tiene un enfoque amplio que incluye una dimensión microsocial, relacionada con la situación personal y la autoresponsabilidad de cada persona, y una perspectiva macrosocial vinculada con responsabilidad pública de implementar las políticas adecuadas que permitan generar oportunidades de envejecimiento activo para las personas mayores (Zamarrón, 2013).

El trabajo social es una ciencia que busca mejorar de la calidad de vida de todas las personas y promover sus potencialidades de manera multidimensional. En esta línea, es una profesión que abandera principios como la justicia social, los derechos humanos, la responsabilidad colectiva y el respeto a la diversidad (FITS, 2014). Es decir, el trabajo social se define por su función de defensa de los derechos humanos de todas las personas y, en el caso que nos ocupa, de las personas mayores.

En el análisis bibliográfico se aprecia esta vinculación explícita entre ambas cuestiones. Muestra de ello son unos objetivos acordes con las dimensiones del concepto de envejecimiento activo, unos principios inspiradores coherentes y en muchos casos compartidos, e incluso una metodología de acción propuesta con factores comunes. En esta línea, varios autores apuntan al trabajo social como una disciplina clave para la promoción de un envejecimiento óptimo (De León, Lévy, Fernández, Ballesteros, 2015; Min, 2013; Ludí, 2012; Barbosa et al., 2015; FernándezGarcía y De León, 2013; Amy, 2014; Ahmed, 2013; Tiernes, 2011; Martín, 2012; García, 2012).

Podemos entender que el trabajo social considera las necesidades derivadas del proceso de envejecimiento de la persona e interrelaciona diferentes perspectivas de ellas. Así, aspectos como autonomía, salud, vivienda, personal, o relaciones familiares y sociales, entre otros, son tenidos en cuenta por los profesionales del trabajo social en sus intervenciones profesionales. Esta dimensión holística es una fortaleza para acometer programas de promoción del envejecimiento activo que requieren un enfoque integrador y multidimensional. Este concepto (OMS, 2002) es un elemento integral, conformado por factores sociales, personales, económicos, de sanidad y servicios sociales, conductuales, de género y culturales. Esta idea refuerza la hipótesis de que los profesionales de trabajo social tienen un perfil profesional adecuado para el diseño y desarrollo de programas e iniciativas que promuevan el envejecimiento activo. Posteriormente abordaremos cómo las dimensiones y los determinantes del envejecimiento activo se reflejan en objetivos profesionales.

Los autores justifican el perfil profesional del trabajo social para promover acciones de envejecimiento activo de forma eficaz. En esta línea podemos afirmar, en palabras de Min, que:

Social workers equipped with many abilities and resources can provide older adults at risk with the opportunities for participation and activities. This way, older adults can be protected from any ill-consequences of the economic crisis and health in- equalities. Therefore, social workers with necessary training and abilities are perfectly positioned to make a difference for older adults at risk. It is a great time that social workers in Spain step up and lead their efforts to become a critical link for the vulnerable seniors. ${ }^{2}$ (Min, 2013: 85)

Así, la atención a la vulnerabilidad es un elemento que se reitera en la bibliografía; los autores manifiestan la preocupación por estos colectivos en los que a la edad se añaden otros criterios de vulnerabilidad, como pueden ser situaciones de exclusión social, de dependencia, o de soledad no deseada, entre otras. Ponen en valor la necesidad de que estos profesionales trabajen en la promoción de la inclusión social y faciliten el acceso y la participación de las personas mayores en los diversos espacios, como pueden ser los sociales, educativos y culturales (Min, 2013; Ludi, 2012; Nieto, 2014).

Los derechos fundamentales recogidos en la Declaración Universal de los Derechos Humanos (ONU, 1948) han de ser barrera infranqueable en las intervenciones sociales. Esto se ha de trasladar a los

\footnotetext{
2 "Trabajadores sociales equipados con muchas habilidades y recursos pueden proporcionar a los adultos mayores en riesgo opor tunidades de participación y actividades. De esta manera, los adultos mayores pueden ser protegidos de las consecuencias negativas de la crisis económica y las desigualdades de salud. Por lo tanto, los trabajadores sociales con la capacitación y las habilidades necesarias están perfectamente posicionados para marcar una diferencia para los adultos mayores en riesgo. Es un buen momento para que los trabajadores sociales en España intensifiquen y lideren sus esfuerzos para convertirse en un enlace crítico para las personas mayores vulnerables" (traducción de la autora).
} 
objetivos, principios y funciones de los profesionales del trabajo social en su práctica profesional. Esta conexión con los derechos humanos se recoge en diversos documentos, como la Declaración Global de Principios Éticos de Trabajo Social (2018), el Código Deontológico del Trabajo Social (2012) y el Estatuto de la Profesión de Diplomado en Trabajo Social (2001), que posicionan a este profesional como agente social de cambio y garante de los derechos de las personas.

\subsection{Determinantes del envejecimiento activo y objetivos del trabajo social}

Para profundizar en la relación existente entre trabajo social y envejecimiento activo nos vamos a centrar en diferentes dimensiones. La primera de ellas hace referencia a los factores determinantes del envejecimiento activo (Imserso; 2001). Para ello relacionamos estos con los objetivos de intervención de los profesionales de Trabajo Social con las personas mayores. Vamos a considerar cada uno de los determinantes como grandes ejes en torno a los cuales se agrupan las metas profesionales.

Son varios los autores que ponen la mirada en estas relaciones, entre los determinantes del envejecimiento activo y los objetivos del trabajo social. Así se refleja en el análisis bibliográfico. Aparecen objetivos tan relevantes como mejorar la calidad de vida, promover la dignidad y autonomía, y favorecer la integración social y la participación de las personas mayores (Barbosa et al., 2015; Foster y Boxall, 2015; Marsillas, 2016; Amy, 2014;
Salmerón, 2012). Señalan que las intervenciones profesionales del trabajo social han de atender las necesidades de diferente naturaleza que conlleva el proceso de envejecimiento, como salud, actividad, independencia y participación, superando un concepto de intervención relacionado únicamente con ocio y tiempo libre.

\subsubsection{Factores sociales}

En primer lugar, contamos con los factores sociales. Estos tienen un gran peso para los profesionales del trabajo social, como se refleja en la bibliografía consultada, que está integrado por variables como educación, alfabetización, derechos humanos, apoyo social, prevención de la violencia y el abuso (Imserso, 2001).

La atención a las personas mayores se ha abordado con frecuencia, atendiendo a la especial protección en base a la vulnerabilidad de estos, derivada de déficits y limitaciones. Sin embargo, autores como Ludi, (2012), De la Villa, (2017) o Salmerón (2012) establecen como objetivo promover una perspectiva de derechos diferenciada de la de tutela, en la que se ponga el acento en la garantía de derechos. En esta línea la Estrategia Nacional de Personas Mayores para un Envejecimiento Activo y Buen Trato 20182021 recoge una línea de actuación relativa a la no discriminación, igualdad de oportunidades, atención a situaciones de fragilidad y vulnerabilidad. Así Ludí (2012) establece que los profesionales del trabajo social han de trabajar promoviendo los derechos de las personas frente a un proteccionismo de los diversos sistemas.

Figura 1. Determinantes del envejecimiento activo

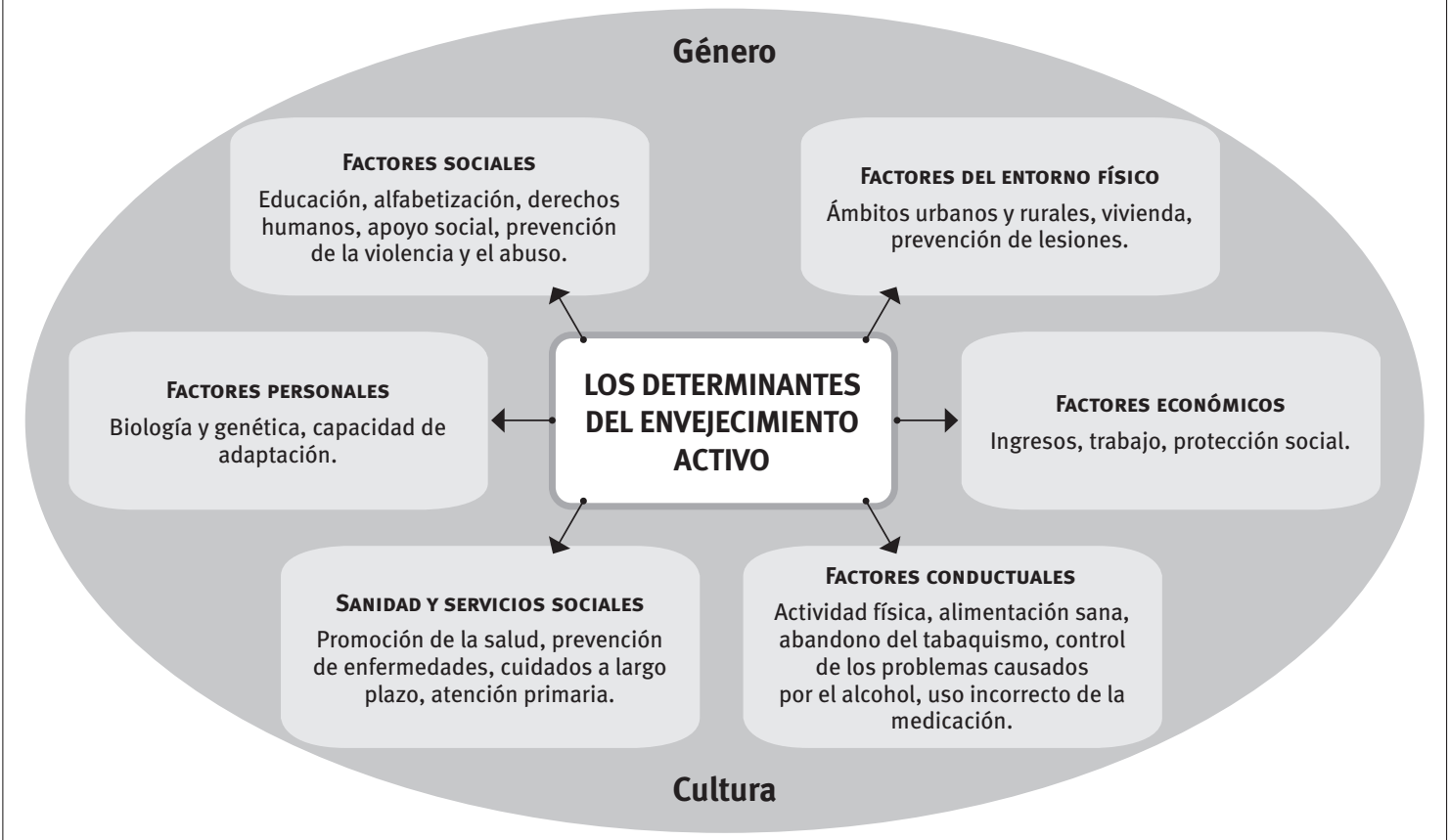

Fuente: Imserso (2001). 
Este planteamiento implica el reconocimiento a la persona mayor como sujeto de derechos y no solo de protección derivada de la inferioridad de condiciones. Por ende, esto implica considerar las oportunidades y fortalezas y no solo los déficits y limitaciones, así como repensar a la persona mayor como agente activo y productivo social y emocionalmente.

Este paradigma de la persona mayor como agente participativo y activo se refleja en aquellas que desarrollan acciones de voluntariado, que atienden a otras personas en situación de dependencia, sean personas mayores o no, que colaboran en acciones intergeneracionales, que desempeñan labores de cuidado que posibilitan la conciliación laboral y familiar; constituyen realidades en muchos casos ignoradas e invisibilizadas que es preciso dimensionar y poner en valor para contribuir a eliminar los estereotipos negativos existentes en torno a las personas de edad avanzada y reforzar al mismo tiempo el paradigma positivo del envejecimiento.

Un segundo objetivo que ubicamos en factores sociales a pesar de que excede la dimensión social y de servicios sociales está relacionado con promover la inclusión social. Se concreta en enunciados como, por ejemplo, impulsar programas de no exclusión social (Barbosa et al., 2015; Ludi, 2012). La inclusión social entendida como la plena participación de la persona en las estructuras participativas y de poder constituye un aspecto clave a ser considerado. Por ello, desde el punto de vista de los autores, los profesionales del trabajo social han de orientar sus esfuerzos a que las personas mayores participen, decidan e intervengan en las diferentes estructuras sociales, y no solo aquellas dirigidas exclusivamente a las personas mayores, que en muchos casos las aíslan y refuerzan el edadismo social.

Mejorar las relaciones familiares y sociales de las personas mayores es otro de los objetivos de los profesionales del trabajo social que está relacionado con el apoyo social. Así, Ludi (2012) y De León et al. (2015) proponen explícitamente que los profesionales del trabajo social establezcan objetivos relacionados con la mejora relacional desde un nivel familiar y social. Esto influye directamente en la prevención de la soledad no deseada, ya que cuanto más sólida y estable es la red familiar y social de una persona mayor, menor es la soledad percibida por ella. Iniciativas como Siempre Acompañados, impulsada por Fundación la Caixa, promueven la intervención social con personas mayores que sienten soledad no buscada con el objetivo de reducir esta y favorecer el establecimiento de relaciones familiares y sociales satisfactorias.

Por último, relacionado con la cultura y alfabetización, diversos autores señalan la importancia de promover el aprendizaje a lo largo de la vida y destacan el carácter preventivo del desarrollo de acciones formativas (Cordero del
Castillo, 2012; Salmerón, 2012; De León et al., 2015; Aparicio, 2013). Al abordar los recursos sociales para promover el envejecimiento activo anotan los cursos formativos, de alfabetización, las universidades para mayores y los programas de estimulación cognitiva como espacios a desarrollar por parte de los profesionales del trabajo social en la promoción del envejecimiento activo (De León et al., 2015).

\subsubsection{Sanidad y servicios sociales}

Otro determinante del envejecimiento activo lo constituye el relacionado con sanidad y servicios sociales. Este es significativo para el trabajo social y evidencia de ello es la diversidad de objetivos y metas profesionales relacionadas con él.

El objetivo que aparece con mayor frecuencia al abordar la intervención de los profesionales del Trabajo Social se relaciona con promover la atención psicosocial y el asesoramiento social (Fernández-García y De León, 2013; Tiernes, 2011; Amy, 2014). Así, la relación de ayuda se constituye como un elemento clave que va a potenciar el empoderamiento y desarrollo de las personas mayores:

Los trabajadores sociales no solamente gestionan los recursos para atender la demanda, sino que también pueden complementar esta función (principalmente burocrática) con una intervención más existencialista y fenomenológica, orientando los proyectos vitales a la búsqueda de acontecimientos agradables y beneficiosos para afrontar esta etapa con ilusión, actividad, dinamismo y entusiasmo. (Fernández-García y de León, 2013: 95)

El profesional del trabajo social, al igual que otros profesionales, tiene su mirada puesta en los factores de riesgo y vulnerabilidad de las personas, por lo que puede realizar intervenciones para atender socialmente a personas mayores vulnerables. Incrementar los factores de protección y disminuir los de riesgo, tales como pobreza, discapacidad o género, entre otros, constituirían objetivos profesionales de este colectivo (Min, 2013; Ludi, 2012; Marshall y Altpeter, 2005).

De la mano del anterior aparece otro objetivo, el de enlazar de las necesidades sociales con los recursos existentes (De León et al., 2015; McDonald, 2010; Fernández-García y de León, 2013; Amy, 2014). Apunta a que los profesionales han de ser capaces de realizar valoraciones de las necesidades sociales, sanitarias, personales y educativas de las personas mayores y sus familias, así como estar informados sobre los diferentes recursos existentes en este ámbito: residencias, centros de día, centros de participación, programas de voluntariado, etc. Al tomar como referencia las necesidades de cada persona o grupo tratará de enlazar y darles respuesta a través de la asignación de recursos. 
En definitiva, emergen diferentes metas profesionales relacionadas con los recursos sociales, desde promoverlos y movilizarlos, hasta acercar y enlazar necesidades y los recursos sociales. Este profesional, al estar en contacto con las necesidades sociales de los individuos, grupos y comunidades, tiene capacidad para realizar el ajuste oportuno entre todos ellos. Como apuntan de León et al. cuando citan a McDonald:

The social worker should seek efficient ways to enrich older people's social environment, thereby encouraging them to use their memory: for example, by helping them improve relationships with their families and friends, encouraging them to participate in social activities centers (cultural lectures, guided tours, hiking, dancing, and so on), proposing new challenges (for example, enrolling in university courses), and describing available resources (such as domiciliary services, assistive technology, day services, residential service, and volunteering. ${ }^{3}$ (De León et al., 2015: 187)

Así, resulta de interés que las personas mayores puedan acceder fácilmente a los profesionales de trabajo social y que estos puedan desempeñar funciones de apoyo psicosocial, orientación e información, entre otras, para mejorar la calidad de vida de aquellas.

\subsubsection{Factores económicos}

En cuanto a los factores económicos, es fundamental abordar los ingresos, el trabajo y la protección social (Imserso, 2001). Así, garantizar la seguridad económica y minimizar las situaciones de pobreza son objetivos principales de las intervenciones de los profesionales de trabajo social. Atender la cobertura de las necesidades básicas, incluyendo la seguridad económica con la gestión de recursos oportunos, es una meta profesional tal y como recogen Ludi (2012) y Min (2013).

\subsubsection{Factores personales y conductuales}

Otros de los determinantes que forman parte del concepto de envejecimiento activo son los factores personales y conductuales. Los primeros hacen referencia a la biología, a la genética y a la capacidad de adaptación de la persona, y los segundos, los conductuales, abordan el autocuidado y los estilos de vida saludable (Imserso, 2001; OMS, 2002;). Así, potenciar las capacidades de los usuarios a

3 "El trabajador social debería buscar maneras eficientes de enriquecer el entorno social de las personas mayores, alentándolos a utilizar su memoria: por ejemplo, ayudándoles a mejorar las relaciones con sus familias y amigos, alentándolos a participar en centros de actividades sociales (conferencias culturales, visitas guiadas, caminatas, bailes, etc.), proponiendo nuevos retos (por ejemplo, matricularse en cursos universitarios) y describiendo los recursos disponibles (como servicios domiciliarios, asistencia tecnología, servicios diurnos, servicio residencial y voluntariado)". través del apoyo psicosocial es uno de los objetivos profesionales de estas áreas para que puedan desarrollar de forma eficaz actividades físicas y alimentación sana, entre otras. Por otro lado, surgen metas profesionales de promoción de espacios y acciones que permitan desarrollar acciones formativas con esta finalidad (Fernández-García y de León, 2013; Ludi, 2012).

\subsubsection{Factores del entorno físico}

La última dimensión abordada se corresponde con los factores físicos. Se trata de cuestiones relacionadas con ámbito rural y urbano y la vivienda (Imserso, 2001) Una cuestión interesante que aparece es la promoción por parte de este profesional de ciudades amigables que favorezcan el envejecimiento de las personas mayores en su entorno (Marshall y Altpeter, 2005).

\subsubsection{Género y cultura}

El género y la cultura son considerados por la OMS (2002) como determinantes transversales que influyen directamente en el resto de los determinantes. Por un lado, el género modifica la manera de envejecer, ya que implica un acceso desigual a recursos económicos, trabajo y servicios, siendo las mujeres un grupo especialmente vulnerable. Por otra parte, las políticas y programas que se implanten deben respetar las culturas y creencias del lugar donde se instauren y promover la igualdad de género (Marina y Jonás, 2012; Cordero del Castillo, 2012; Nieto, 2014; Salmerón, 2012). Tal y como recoge Salmerón (2012), las mujeres son más proclives a sufrir pobreza y discapacidad o enfermedad, y son más dependientes de servicios sociales y sanitarios.

Así, la cultura, los valores y las tradiciones influyen en la visión que se tiene de las personas mayores y en los procesos de envejecimiento (Nieto, 2014; Bosch et al., 2009). Los objetivos han de respetar las culturas y creencias del lugar donde se interviene; en esta línea, los autores remarcan la importancia de estudiar e investigar el contexto físico y cultural (Bellido, 2013; Ahmed, 2013; Bosch et al., 2009).

\subsection{Otros objetivos/otras consideraciones}

Existe una serie de metas y objetivos de las intervenciones profesionales que son comunes, ya que afectan a todas las áreas y determinantes del envejecimiento activo. Estas cuestiones se detallan de manera más concisa a continuación.

La primera hace referencia a mejorar la calidad de vida de individuos, grupos y comunidades. Esta cuestión aparece en los diferentes textos y es uno de los objetivos más frecuentes de las intervenciones profesionales del trabajo social que tienen en las 
personas mayores su ámbito de actuación. Así, los autores entienden que los profesionales han de tener en su horizonte cuestiones como promover la calidad de vida y la dignidad de los mayores (Ludi, 2012; Fernández-García y de León, 2013; Redondo, Gonzalo, Veiguela, Bacariza y Otero, 2012; Barbosa et al., 2015; Tiernes, 2011; Amy, 2014; Ahmed, 2013).

Una segunda cuestión que aparece formulada como objetivo del trabajo social en relación con el envejecimiento activo está vinculada a la planificación y la evaluación. Así, se habla de desarrollar políticas públicas y proyectos de no exclusión de las personas mayores en la sociedad promoviendo el envejecimiento activo (Barbosa et al., 2015; Ludi, 2012) y de que junto con el desarrollo de los programas se deben desplegar estrategias de evaluación de las políticas públicas con el fin de valorarlas y conocer si son eficaces para conseguir un envejecimiento activo real de la población, como metas que aparecen en las intervenciones profesionales. Así apunta Barriga (2010, citado en Barbosa et al., 2015).

Cierto es que la planificación y la evaluación son base de acciones eficaces. Las políticas sociales y públicas constituyen el motor de cambio de la sociedad y estas han de incorporar los objetivos aquí señalados.

Investigar también constituye un objetivo que ha de contemplarse. Así, García (2012) o Bosch et al. (2009) establecen como objetivo profesional indagar científicamente en aspectos tales como la participación de las personas mayores en diversas actividades y su influencia en la calidad de vida.

Como observamos en la relación de los determinantes del envejecimiento activo y los objetivos de los profesionales de trabajo social, todas las áreas en mayor o menor medida tienen objetivos de intervención profesional, lo que ampara la hipótesis de que el trabajo social, como disciplina holística y multidimensional, es clave para la promoción del envejecimiento activo.

De la mano de los objetivos y metas profesionales caminan los principios que van a guiar e inspirar la praxis de los profesionales del trabajo social. Estos principios van a indicar cómo se deberían implementar las intervenciones que promuevan el envejecimiento activo.

\subsubsection{Principios del envejecimiento activo y del trabajo social}

Los principios inspiradores del envejecimiento activo son apuntados por distintos autores (Walker, 2002; Foster y Boxall, 2015; Foster y Walker, 2015; Marsillas, 2016; Cordero del Castillo, 2012). De forma particular, Marsillas presenta (2016) la compilación del abordaje de dichos principios como elemento de ayuda para implementar las prácticas de envejecimiento activo: la actividad, la inclusión, la prevención, la solidaridad intergeneracional, la participación y empoderamiento, la diversidad nacional y cultural, la flexibilidad y el reconocimiento de derechos y obligaciones (Walker, 2002; Foster y Walker, 2015).

Para conocer si existe vinculación entre los principios que guían ambos elementos, tomamos como referencia la reciente Declaración de Principios Éticos del Trabajo Social elaborada por la FITS (2018). Esta declaración de principios sirve como marco general para que estos profesionales trabajen en aras de la ética y de la integridad profesional. Observamos, al igual que en el caso de los objetivos, la relación entre los principios del Envejecimiento Activo con los principios deontológicos de esta disciplina. Así, en la Figura 2 se presenta la coincidencia explícita de estos.

Figura 2. Vinculación de los principios del envejecimiento activo y los principios del trabajo social

\begin{tabular}{|c|c|c|}
\hline $\begin{array}{l}\text { Principio de } \\
\text { envejecimiento activo }\end{array}$ & Principio de trabajo social (FITS, 2018) & Autores \\
\hline Actividad & $\begin{array}{l}\text { Derecho a la participación para el desarrollo de la autoestima y las capacidades } \\
\text { de las personas (5). }\end{array}$ & $\begin{array}{l}\text { Walker, 2002; Marsillas, } \\
2016 .\end{array}$ \\
\hline $\begin{array}{l}\text { Inclusión de todas las } \\
\text { personas mayores }\end{array}$ & $\begin{array}{l}\text { Promoción de la justicia social respetando la diversidad, respetando las } \\
\text { diferencias individuales y facilitando el acceso a todo tipo de recursos (3.3). }\end{array}$ & $\begin{array}{l}\text { OMS, 2002; Walker, 2002, } \\
\text { 2006; Marsillas, } 2016 .\end{array}$ \\
\hline Prevención & & $\begin{array}{l}\text { OMS, 2002; Walker, 2002, } \\
\text { 2006; Marsillas, } 2016 .\end{array}$ \\
\hline $\begin{array}{l}\text { Solidaridad } \\
\text { Intergeneracional }\end{array}$ & Construcción de redes de solidaridad (3.5). & $\begin{array}{l}\text { Walker, 2002, 2006; } \\
\text { Marsillas, } 2016 .\end{array}$ \\
\hline $\begin{array}{l}\text { Derechos y } \\
\text { obligaciones }\end{array}$ & Promover de los derechos humanos (2) y el derecho a la autodeterminación (4). & $\begin{array}{l}\text { Walker, 2002, } \\
\text { 2006; Marsillas, } 2016 .\end{array}$ \\
\hline $\begin{array}{l}\text { Participación y } \\
\text { empoderamiento }\end{array}$ & Derecho a la autodeterminación (4) y a la participación (5). & $\begin{array}{l}\text { Walker, 2002, } \\
\text { 2006; Marsillas, } 2016 .\end{array}$ \\
\hline $\begin{array}{l}\text { Diversidad nacional y } \\
\text { cultural }\end{array}$ & Promoción de la justicia social respetando la diversidad (3). & $\begin{array}{l}\text { Walker, 2002, } \\
\text { 2006; Marsillas, } 2016 .\end{array}$ \\
\hline $\begin{array}{l}\text { Flexibilidad de la } \\
\text { aproximación }\end{array}$ & $\begin{array}{l}\text { Autodeterminación en la toma de decisiones }(4) \text { y tratar a la persona como } \\
\text { un todo reconociendo las dimensiones biológicas, psicológicas, sociales y } \\
\text { espirituales ( } 7 \text {. }\end{array}$ & Foster y Walker, 2015. \\
\hline
\end{tabular}

Fuente: Elaboración propia. 
A la vista de estos elementos, podemos señalar varias cuestiones de interés que se desprenden de los principios de ambos conceptos y nos apuntan un camino a seguir. En primer lugar, las personas mayores han de considerarse como sujetos de derechos y los profesionales del trabajo social como promotores y garantes de los derechos humanos que acompañan a las personas mayores en este proceso de transformación vital y personal.

En segundo término, existe la necesidad de inclusión de todas las personas mayores en las políticas de envejecimiento activo y la atención a todas las personas mayores con independencia de su edad, nivel de dependencia, nacionalidad, situación económica, o de salud, entre otras circunstancias.

Una tercera cuestión que cabe destacar responde a qué principios de actividad, participación y empoderamiento son elementos compartidos, ya que los profesionales del trabajo social tienen como misión desarrollar la autoestima y las capacidades de las personas, promoviendo su plena implicación y participación en todos los aspectos de las decisiones $\mathrm{y}$ acciones que afectan sus vidas.

Un cuarto aspecto apunta la importancia de la solidaridad, tanto hacia otras personas mayores como a grupos vulnerables, personas con capacidades diferentes y colectivos en situación de exclusión social, entre otros, como forma de generar sociedades más inclusivas, que es uno de los principios del trabajo social.

La siguiente cuestión estaría relacionada con la importancia para el trabajo social de la promoción de la justicia social a través de la lucha contra la discriminación, el respeto a la diversidad nacional y cultural, y el acceso a recursos equitativos, la lucha contra políticas injustas y la construcción de la solidaridad.

Un aspecto más a destacar se basa en el reconocimiento de un enfoque integral y flexible del envejecimiento activo (Foster y Walker, 2015). Esto, por un lado, implica la consideración de las personas como un todo y la promoción del reconocimiento de las dimensiones biológicas, psicológicas y espirituales de las personas (FITS, 2018). Por otra parte, conlleva superar las intervenciones aisladas y la parcialización de las personas.

A la vista de lo anterior, observamos una correlación entre los principios del envejecimiento activo (Walker, 2002; Foster y Walker, 2015; Marsillas, 2016) y los recogidos por la FITS: la dignidad, la independencia, la autorrealización, la actividad y la solidaridad aparecen en ambos documentos como guías de la práctica profesional. Podemos apuntar que el trabajo social establece el reconocimiento de la dignidad inherente a cada ser humano a través del respeto a todas las personas y del desafío las creencias y acciones de aquellos individuos que se devalúan o estigmatizan a ellos mismos o a otras personas (FITS, 2018: 1).

En la misma línea, otros autores incorporan nuevas cuestiones que complementan los principios anteriormente señalados, como el reconocimiento de las labores que realizan las personas mayores en el medio familiar (Cordero del Castillo, 2012), siendo este asunto de especial relevancia por su contribución a reforzar el paradigma positivo de las personas mayores en el que se las percibe desde un enfoque constructivo de aportación social y comunitaria.

Hemos señalado los principios en los que apreciamos una coincidencia clara; sin embargo, en la Declaración de Principios Éticos del Trabajo Social se ponen en valor otras cuestiones que consideramos relevantes y que no aparecen vinculadas de forma explícita con el envejecimiento activo, como son el respeto a la confidencialidad y privacidad de todas las personas, un uso ético de la tecnología y las redes sociales y la integridad profesional con el cumplimiento de esta declaración de principios éticos. Es interesante tener en cuenta estos aspectos en las intervenciones con personas mayores, ya que pueden ayudar en el desempeño de la práctica profesional de forma que contribuyan a la mejora de la calidad de vida de estas.

En conclusión, podemos señalar que los principios que guían el envejecimiento activo y el trabajo social tienen unos puntos de unión significativos que relacionan ambas cuestiones de una forma clara y directa. Esto apunta a la existencia de un marco común entre el trabajo social y el envejecimiento activo. A continuación, vamos a presentar los resultados de la búsqueda bibliográfica que abordan los niveles de intervención de estos profesionales.

\subsubsection{Niveles de atención de trabajo social en materia de envejecimiento activo}

El trabajo social con personas mayores cuenta con una gran fortaleza, que consiste en la presencia de este profesional en la mayoría de los servicios y recursos en los que se trabaja con personas mayores. Así, residencias, centros de día, centros de participación activa u hogares de personas mayores y servicios de ayuda a domicilio, entre otros, cuentan en sus plantillas con profesionales del trabajo social como personal clave de sus equipos técnicos. En cada uno de ellos, y atendiendo a diferentes variables como pueden ser la ubicación en un contexto rural o urbano, las necesidades de las personas, la disponibilidad horaria del profesional, las funciones asignadas por la entidad y otras cuestiones, las intervenciones profesionales van a orientarse y perfilarse a dimensiones individuales, grupales y/o comunitarias. En este apartado se analizan los niveles de intervención del trabajo social en envejecimiento activo. 
En la revisión bibliográfica aparecen frecuentes referencias al trabajo social individual y comunitario. Así, se constata que en las funciones atribuidas a este perfil profesional resultan claves, principalmente, cuestiones como el apoyo psicosocial, la prestación de recursos sociales o la cobertura de las necesidades básicas, que se realizan de forma prioritaria a través de una atención individual y familiar. Este ámbito se potencia de forma explícita en las intervenciones profesionales, en detrimento en muchos casos del trabajo social grupal o comunitario (Marshall y Altpeter, 2005; Ahmed, 2013; Fernández-García y de León, 2013).

Así, Fernández-García y De León (2013) ponen las palabras de Derezotes sobre el planteamiento del método de gestión de casos como metodología eficaz en niveles individual y/o familiar y apunta que:

El modelo de intervención que mejor se adapta a la consecución de un envejecimiento activo y saludable mediante la movilización de apoyos sociales es el denominado Modelo de Gestión de Casos, porque intenta asegurar una serie de servicios de forma eficaz, eficiente, razonada y coordinada para el cumplimiento de las necesidades. La finalidad del modelo es facilitar información sobre nuevas oportunidades, acercando los recursos sociales disponibles a la persona con el fin de colaborar en el desarrollo del bienestar y la mejora de la calidad de vida del usuario. (Fernández-García y de Leon, 2013: 92)

En segundo lugar, el trabajo social comunitario tiene también una gran presencia en las referencias bibliográficas consultadas. Autores como Barbosa et al. (2015), Ahmed (2013) o Marshall y Altpeter (2005) coinciden en la importancia de que los profesionales del trabajo social planifiquen y ejecuten acciones comunitarias para la promoción del envejecimiento activo. Proponen espacios comunitarios en los que colaboran personas de diferentes edades y entidades, superando espacios y acciones dirigidas única y exclusivamente a personas de edad avanzada.

Cuestiones como la promoción de la participación de las personas mayores, el fomento del asociacionismo, el trabajo en red, proyectos intergeneracionales entre otras, se convierten en elementos clave y compatibles con el ámbito comunitario de los profesionales del trabajo social. En este sentido Barbosa et al. (2015) ponen en valor y reclaman esta dimensión comunitaria. Su defensa se enlaza con el cariz reivindicativo y de defensa de la justicia social propio del profesional del trabajo social. Aluden a la falta de legislación adecuada para las personas mayores y reivindican espacios comunitarios para trabajar con las personas mayores. El siguiente fragmento de Barbosa refleja con claridad las cuestiones apuntadas.

El trabajador social, viendo el panorama de falta de apoyo y limitadas políticas sociales, legislación carente dirigida al mayor y la pobre conciencia social que existe para trabajar con las personas mayores, debería actuar por otras vías. Comenzar a trabajar con el mayor desde la comunidad, trabajando de forma conjunta con otros colectivos de edades, no apartar a las personas mayores en actividades sólo dirigidas a ellos. Aprovechando el apoyo comunitario, seguir la vía más social, organizar bancos del tiempo, sistema de cadena de favores, de forma que se impliquen ambas partes por igual tanto mayores, como adultos, adolescentes, menores, etc., de este modo a la vez de que se conciencia y demuestra que el mayor está tan cualificado para participar como otro rango de edad, se le da al mismo el sentimiento de ser útil para los demás, para sí mismo y para la sociedad. (Barbosa, 2015: 177)

Además de la participación social, la bibliografía consultada pone en valor la importancia del capital humano de las personas mayores, su productividad social en esta dimensión comunitaria (Barbosa et al., 2015; Ahmed, 2013; Marshall y Altpeter, 2005; De León et al., 2015). Por un lado, resaltan las aportaciones a la sociedad a través del voluntariado, acciones intergeneracionales, acciones solidarias entre otras; por otro, destacan el potencial del trabajo social para intervenir desde el ámbito comunitario, poniendo como ejemplo de estas estructuras participativas algunos consejos de personas mayores que tratan de dar voz a las personas mayores que están organizadas en diferentes entidades u organizaciones, posibilitando que puedan decidir acerca de las cuestiones que les preocupan y les atañen (Ahmed, 2013).

Además de estos aspectos, hay que destacar que "el trabajo social comunitario, en base a sus fundamentos teóricos y metodológicos, se postula como un método de intervención social capaz de llevar a buen puerto procesos de empoderamiento comunitario" (Ahmed, 2013: 27), mejorando la calidad de vida de las personas mayores (Barbosa et al., 2015; Ahmed, 2013).

Respecto al nivel de intervención grupal del trabajo social, cabe destacar que son escasas las referencias bibliográficas que vinculan el envejecimiento activo con dicha dimensión. Esta constatación nos lleva a interrogarnos si la invisibilidad o inexistencia del trabajo social grupal en la bibliografía es reflejo de la ausencia de intervenciones profesionales desde este ámbito o de la falta de sistematización de dichas prácticas grupales.

En materia de envejecimiento activo se trabaja desde un nivel individual y comunitario, y los autores reclaman la promoción de acciones grupales y comunitarias por el potencial de estas. En definitiva, se trata de poner en valor la dimensión comunitaria del trabajo social, entendiendo que la cuestión del envejecimiento es una situación estructural que requiere de respuestas globales, comunitarias e integrales. Por ello, el abordaje ha de ir más allá 
de las respuestas a las necesidades concretas e individuales de cada ciudadano.

\section{Retos futuros para la promoción del envejecimiento activo desde el trabajo social}

A la vista de las referencias bibliográficas consultadas que vinculan el trabajo social y el envejecimiento activo aparecen diferentes cuestiones y retos de futuro que posibilitarían un mejor desarrollo de las políticas y planes de envejecimiento activo y mejores intervenciones profesionales.

En primer lugar, el envejecimiento activo ha de ser inclusivo y ha de dirigirse a toda la población mayor. Ha de tener en cuenta a las personas mayores con discapacidad, a las personas en situación de exclusión social o con bajos recursos económicos, a aquellas que sufren soledad no deseada, y a las que residen en contextos rurales o en centros residenciales. Se trata de personas o colectivos que presentan unas necesidades específicas concretas requieren intervenciones adaptadas desde la perspectiva del trabajo social.

En línea con lo anterior, y considerando la multidimensional del concepto de envejecimiento activo, las acciones promotoras de este han de implementarse desde los diferentes sistemas de protección de la salud, servicios sociales, justicia o educación, que respondan a las diferentes necesidades de la persona. Es decir, las intervenciones han de atender a los criterios de atención integral y abordar especialmente las necesidades psicosociales de la persona, de individualización, considerar las características específicas de cada persona y de su ecosistema, trabajo en red y coordinación.

Por otra parte, se torna imprescindible poner en valor los tres niveles de intervención individual, grupal y comunitaria. Tener la mirada abierta a proyectos grupales y comunitarios posibilita aprovechar al máximo el potencial de estos espacios de intervención que pueden ser más eficaces que los niveles individuales.

Es fundamental que se cuente con la opinión de las personas mayores, si es posible de forma cualitativa, en los procesos de toma de decisiones y que las políticas y programas no sean planificadas exclusivamente por políticos y por técnicos al margen de los principales protagonistas, las personas mayores. Los profesionales del trabajo social, como especialistas en escucha activa, tienen herramientas y habilidades para conocer a las personas y favorecer su participación.

Así, se torna imprescindible que las personas mayores sean agentes activos de su propio desarrollo, han de decidir y opinar acerca de las cuestiones que les afectan en los diferentes ámbitos de su vida: salud, vivienda, educación, jubilación, etc. Las políticas sociales orientadas a las personas mayores han de ser realizadas por las propias personas mayores, acompañadas en el proceso de envejecimiento por técnicos con perfil profesional de trabajo social.

Estos retos y mejoras han de incluir a los profesionales e incorporar en los estudios de grado -en este caso, de trabajo social- formación específica sobre personas mayores, envejecimiento activo, atención a situaciones de dependencia, etc., dotando a los profesionales de competencias y habilidades específicas para trabajar con este grupo de población.

Todas estas propuestas planteadas por los autores nos marcan un camino en las intervenciones para promover el envejecimiento activo. Unas más sencillas de alcanzar y otras quizás más complejas, pero todas ellas necesarias para responder a las necesidades generadas por la oportunidad demográfica en la que estamos inmersos. Esto nos invita a mirar de frente y no girar la vista, poniendo en valor el potencial de esta profesión para trabajar al lado de las personas. 
AHMED MOHAMED, K. y ARIAS ASTRAY, A. (2012): Asociacionismo y calidad de vida en el marco del envejecimiento activo: posibilidades del trabajo social comunitario, Tesis doctoral, Universidad Complutense de Madrid.

APARICIO ALONSO, C. (2013): Educación y envejecimiento activo: una experiencia comunitaria, Universidad de Alcalá.

AMY RESTORICK, R. (2014): The Influence of Social Engagement on Changes in Quality of Life over Time for Older Adults Living in Senior Housing, The Ohio State University College of Social Work.

BARBOSA L., FERNÁNDEZ R. y RODRÍGUEZ S. (2015): "Envejecimiento Activo", en SARASOLA SÁNCHEZ-SERRANO, J. L., ANTOLINES DOMÍNGUEZ, I. y NÚÑEZ GÓMEZ, J.C. (2015): Trabajo Social y Servicios Sociales para personas mayores, Sevilla, Universidad Pablo Olavide, pp.169-185.

BELLIDO, A. y CANALES, G. (2013): Envejecer viviendo, envejecimiento activo en la población jubilada de la Vega Baja de Alicante, Universidad de Alicante.

BOLETÍN OFICIAL DEL ESTADO (2006): Ley 39/2006, de 14 de diciembre, de Promoción de la Autonomía Personal y Atención a las personas en situación de dependencia, 〈https://www.boe.es/buscar/ act.php?id=BOE-A-2006-21990 .

BOSCH, P.M., GÓMEZ, A., y FERRER, B.S. (2009): “LoS Grandes Olvidados: Las Personas Mayores en el Entorno Rural", en Psychosocia llntervention, 18 (3), pp. 269-277, <https://doi-org.umbral. unirioja.es/10.5093/in2009v18nza7〉. en Trabajo Social/Asistente Social, «http:// www.cgtrabajosocial.com/app/webroot/ files/jaen/files/estatuto $\% 20$ de $\% 20 l a \% 20$ profesion $\% 20$ de $\% 20$ Diplomado $\% 20 e n \% 20$ Trabajo\%20Social.pdf〉.

- (2012): Código Deontológico de Trabajo Social, «http:// www.consejotrabajosocialcyl.org/codigo deontologico_2012.pdf〉.

CORDERO DEL CASTILLO, P. (2012): “Año Europeo del Envejecimiento Activo y la Solidaridad Intergeneracional", Humanismo y Trabajo Social, no 11 , pp. 101-117.

DE LEÓN, L.P., LÉVY, J.P., FERNÁNDEZ, T. y BALLESTEROS, S. (2015): "Modeling Active Aging and Explicit Memory: An Empirical Study", en: Health and Social Work, vol. 40, no 3 , pp. 183-190, <https:// doi.org/10.1093/hsw/hlv044'.

FERNANDEZ-GARCIA, T. y PONCE DE LEÓN ROMERO, L.: "Envejecimento activo: Recomendaciones para la intervencion social desde el modelo de gestion de casos", Portularia, 13(1), pp. 87-97.

JIMÉNEZ, M. (2017): “Programas intergeneracionales y participación social: La integración de los adultos mayores españoles y latinoamericanos en la comunidad", Universitas Psychologica, vol. 16, nn 1, 〈https://doi.org/10.11144/ Javeriana.upsy16-1.pips〉.

FEDERACIÓN INTERNACIONAL DE TRABAJADORES SOCIALES (2018): Declaración Global de Principios Éticos de Trabajo Social, <https://www.ifsw.org/es/ declaracion-global-de-los-principios-eticos-deltrabajo-social/s.

- (2014): Definición del Trabajo Social, 〈https://www.ifsw. org/what-is-social-work/global-definitionof-social-work/definicion-global-del-trabajosocial/s. 
FERNÁNDEZ-GARCÍA, T., y DE LEÓN ROMERO, L. (2013): "Envejecimiento Activo: Recomendaciones para la intervención social desde el modelo de gestión de casos", Portularia, 13(1), pp. 87-97.

FOSTER, L. y BOXALL, K. (2015): “People with learning disabilities and 'active ageing", British Journal of Learning Disabilities, 43(4), pp. 270-276, <https://doi-org.umbral.unirioja.es/10.1111/ bld.12144>

FOSTER, L. y WALKER, A. (2015): Active and successful aging: A European policy perspective, The Gerontologist, 55, pp. 83-90, <https://doi. org/10.1093/geront/gnuo28>.

GARCíA, G. (2012): “Conectados. Envejecimiento Activo", en CONSEJO GENERAL DE TRABAJO SOCIAL, Servicios Sociales y Política Social, Envejecimiento Activo, Madrid, 2012, Consejo General de Trabajo Social, no 98, 1, pp. 53-66.

INSTITUTO DE MIGRACIONES Y SERVICIOS SOCIALES (2017): Estrategia Nacional de Personas Mayores para un Envejecimiento Activo y Buen Trato 2018-2021, Madrid, Imserso, 〈https://www.fundadeps.org/recursos/ documentos/844/02EFE9D3.pdf〉.

- (2001): Boletín sobre el envejecimiento: perfiles y tendencias, 2001, 4 y 5, Madrid, Observatorio de Personas Mayores, 〈http://www.imserso. es/InterPresent1/groups/imserso/documents/ binario/boletinopm4y5.pdf`.

LUDI, M. (2012): “Trabajo Social y 'envejecimiento activo' en Argentina”, CONSEJO GENERAL DE TRABAJO SOCIAL, Servicios Sociales y Política Social, Envejecimiento Activo, Consejo General de Trabajo Social, no 98, 1, pp. 129-142.

MARINA, L. y JONAS, L. (2012): “Active Ageing and successful ageing as explicative models of positive evolutions to elderly people", en Scientific Annals of the "Al. I. Cuza University, lasi, Sociology \& Social Work, vol. $5, \mathrm{n}-1$, pp. 79-91.

MARSHALL, V.W. y ALTPETER, M. (2005): “Cultivating social work leadership in health promotion and aging: Strategies for active aging interventions", Health \& Social Work, 30, pp. 135-144, 〈https:// doi.org/10.1093/hsw/30.2.135'.

MARSILLAS, S. y VARELA, J. (2016): Desarrollo y validación de un Índice Personal de Envejecimiento Activo adaptado al contexto gallego, tesis doctoral, Universidad de Santiago de Compostela.
MARTíN, M. (2012): "Trabajo Social con personas mayores. Teoría y práctica del Trabajo Social gerontológico", Servicios Sociales y Política Social, n-98, 1, pp. 9-34.

MIN, J. (2013): “Health Inequalities and Active Aging: What Can Social Workers Do?", Revista Internacional de Trabajo Social. AZARBE, vol. 2, pp. 81-86

NIETO, M. (2014): “La educación para la salud en las personas mayores como contribución al envejecimiento activo. Una propuesta de intervención", trabajo de fin de grado, Universidad de Valladolid.

ORGANIZACIÓN MUNDIAL DE LA SALUD (2002): "Envejecimiento activo: un marco político", Revista Española de Geriatría y Gerontología, vol. 37, S2, pp. 74-105.

REDONDO, I., GONZALO, M., VEIGUELA, I., BACARIZA, M. y OTERO, P. (2012): “Programa de envejecimiento activo y saludable en Atención Primaria de Salud”, Trabajo social y salud, n- 73, pp.43-48.

SALMERÓN, J. y MARTÍNEZ, S. (2012): La percepción del envejecimiento desde la perspectiva de las mujeres mayores usuarias de centros sociales de la Comarca del valle del Ricote de la Región de Murcia. Sus implicaciones para la educación, tesis inédita, Universidad de Murcia, Facultad de Educación.

SÃO JOSÉ, J.M., TIMONEN, V., AMADO, C. y SANTOS, S.P. (2017): "A critique of the Active Ageing Index", Journal of Aging Studies, vol. 40, pp. 49-56, 〈https://doi.org/10.1016/j.jaging.2017.01.001〉.

TIERNES, C. (2011): “El Trabajo Social y la educación social como pilares básicos para la atención y estimulación cognitiva de nuestras personas mayores", Pedagogia I Treball Social, pp. 48 64.

WALKER, A. (2002): "A strategy for active ageing", International Social Security Review, 55(1), pp. 121-139, 〈https://doi.org/10.1111/1468246X.00118>.

WALKER, A. (2006): “Active ageing in employment: its meaning and potential", Asia Pacific Review, 13(1), pp. 78-93, 〈https://doi. org/10.1080/13439000600697621>.

ZAMARRÓN, M.D. (2013): "Envejecimiento activo: un reto individual y social", Sociedad y Utopía. Revista de Ciencias Sociales, nํ- 41, pp. 449-463. 